\title{
Commentary Paper: Stroke in MELAS is a Vasogenic Edema and not Ischemic
}

\author{
Josef Finsterer $^{* *}$, Sinda Zarrouk-Mahjoub ${ }^{2}$ \\ 1. Neurological Department, Krankenanstalt Rudolfstiftung, Messerli Institute, Vienna, Austria. \\ 2. University of Tunis El Manar and Genomics Platform, Pasteur Institute of Tunis, Tunisia.
}

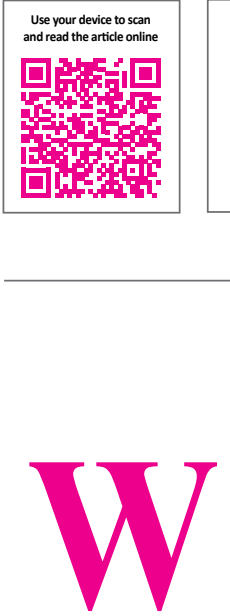

Citation: Finsterer, J., \& Zarrouk-Mahjoub, S. (2020). Stroke in MELAS is a Vasogenic Edema and not Ischemic. Basic and Clinical Neuroscience, 11(3), 379-380. http://dx.doi.org/10.32598/bcn.11.2.1271.1

http://dx.doi.org/10.32598/ben.11.2.1271.1

\section{Commentary}

e read with interest the article by Almasi et al. on a 48 years old female patient with Mitochondrial Encephalomyopathy, Lactic Acidosis, And Stroke-like episodes (MELAS), diagnosed based on the clinical presentation, blood test results, and imaging and muscle biopsy findings (Almasi et al., 2017). We have the following comments and concerns.

We do not agree with the statement that stroke in MELAS is ischemic (Almasi et al., 2017). In the acute stage, Stroke-Like Lesions (SLLs) on Magnetic Resonance Imaging (MRI), the imaging equivalent of a Stroke-Like Episode (SLE), show up as hyperintensity on DiffusionWeighted Imaging (DWI) and mostly as hyperintensity on Apparent Diffusion Coefficient (ADC) maps (ElHattab et al, 2001). Only in the later stages, SLLs may convert from vasogenic edema to non-specific regional abnormalities (El-Hattab et al, 2001). Typical findings on imaging may disappear with the duration of the SLE, therefore, it would be interesting to know how many days after onset of the SLE the MRI had been carried out, and whether the MRI abnormalities completely resolved with the resolution of the clinical manifestations.

As mentioned by the authors, in general, MELAS and Mitochondrial Disorders (MIDs) are considered as mul- tisystem diseases (Finsterer, 2017). The patient obviously manifested with SLEs, seizures, migraine, easy fatigability, lactic acidosis, anemia, and hypoacusis (Almasi et al., 2017). However, in some MELAS patients, additional phenotypic features have been reported, such as short stature, confusion, cognitive impairment, dementia, basal ganglia calcification, ptosis, ophthalmoparesis, visual disturbance, gastrointestinal problems (vomiting and volvulus), renal insufficiency, or neuropathy (Finsterer, \& Frank, 2015). A typical manifestation of MELAS is lactic acidosis. However, the presented patient had respiratory alkalosis despite severely elevated serum lactate, a discrepancy that should be explained.

We do not understand why the patient received Valproic Acid (VPA) after the first seizure. First, a single seizure does not necessarily require treatment with Antiepileptic Drugs (AEDs). Second, it is well established that VPA exerts mitochondrial toxicity (Kim et al., 2011). Surprisingly, fatalities have been reported due to VPA, particularly in patients carrying POLG1 mutations. Thus, it is conceivable that the first SLE in the reported patient was triggered by VPA, unless other triggers for the SLE had been identified. Another therapeutic option for MIDs, particularly mitochondrial epilepsy, is the ketogenic diet and it would be interesting to know whether the patient was subjected to such a low-glucose and fat-rich diet. 
Diagnosing MIDs requires documentation of a pathogenic mtDNA or nDNA variant. This is why we should be informed whether the patient or her first-degree relatives were tested for the most common MELAS mutations. In up to one-quarter of the MID patients, mutations occur spontaneously as de-novo events (Kitamura et al., 2016). Since the parents were clinically unaffected, a de novo mutation is quite likely.

As mentioned by the authors, SLEs are currently well treated with nitric oxide (NO)-precursors, such as L-arginine or L-citrulline (Poulton, Finsterer, \& Yu-Wai-Man, 2017). NO is a potent mediator of cerebral vasodilation and is particularly produced from L-arginine and L-citrulline by the enzymatic activity of NO synthetase. Assuming that SLEs are precipitated by vasoconstriction due to abnormal reactivity of cerebral arteries and that patients with MELAS have low NO serum concentrations, administration of NO-precursors aims at increasing NO serum levels and, consequently mediating vasodilation to treat and prevent SLEs. Thus, it would be of value to know why no NO-precursors were administered but instead, mannitol and dexamethasone. Regarding steroids, it is known that they are associated with both beneficial and detrimental effects (Zhang, Guo, Fang, Jun, \& Shi, 2015).

Overall, we appreciated to read this interesting case, but the report could be more meaningful if the diagnosis would have been confirmed genetically, if NO-precursors would have been administered for the SLE, if possible triggers of the SLE would have been discussed, and if the patient would have been prospectively investigated for subclinical or mildly manifesting abnormalities.

\section{Ethical Considerations}

\section{Compliance with ethical guidelines}

There was no ethical considerations to be considered in this research.

Funding

This research did not receive any specific grant from funding agencies in the public, commercial, or nonprofit sectors.

\section{Authors' contributions}

Both authors c ontributed in designing, running, and writing all parts of the research.

\section{Conflict of interest}

The authors declared no conflict of interest.

\section{Reference}

Almasi, M., Motamed, M. R., Mehrpour, M., Haghi-Ashtiani, B., Haji Akhondi, F., \& Nilipour, Y., et al. (2017). A mitochondrial disorder in a middle age Iranian patient: Report of a rare case. Basic and Clinical Neuroscience, 8(4), 337-41. [DOI:10.18869/ nirp.bcn.8.4.337] [PMID] [PMCID]

El-Hattab, A. W., Almannai, M., Scaglia, F., Adam, M. P., Ardinger, H. H., \& Pagon, R. A., et al. (2001). MELAS. In: M. P. Adam, H. H. Ardinger, R. A. Pagon, S. E. Wallace, L. J H. Bean, \& K. Stephens, et al., (Eds.), GeneReviews ${ }^{\circledR}$ (pp. 1993-2017). Seattle (WA): University of Washington, Seattle. [PMID]

Finsterer, J. (2017). Toxicity of antiepileptic drugs to mitochondria. Handbook of Experimental Pharmacology, 240, 473-88. [DOI:10.1007/164_2016_2] [PMID]

Finsterer, J., \& Frank, M. (2015). Glucocorticoids for mitochondrial disorders. Singapore Medical Journal, 56(2), 122-3. [DOI:10.11622/smedj.2015026] [PMID] [PMCID]

Kim, J. H., Lim, M. K., Jeon, T. Y., Rha, J. H., Eo, H., \& Yoo, S. Y., et al. (2011). Diffusion and perfusion characteristics of MELAS (mitochondrial myopathy, encephalopathy, lactic acidosis, and stroke-like episode) in thirteen patients. Korean Journal of Radiology, 12(1), 15-24. [DOI:10.3348/kjr.2011.12.1.15] [PMID] [PMCID]

Kitamura, M., Yatsuga, Sh., Abe, T., Povalko, N., Saiki, R., \& Ushijima, K., et al. (2016). L-Arginine intervention at hyperacute phase protects the prolonged MRI abnormality in MELAS. Journal of Neurology, 263, 1666-8. [DOI:10.1007/s00415016-8069-4] [PMID] [PMCID]

Poulton, J., Finsterer, J., \& Yu-Wai-Man, P. (2017). Genetic counselling for maternally inherited mitochondrial disorders. Molecular Diagnosis \& Therapy, 21, 419-29. [DOI:10.1007/s40291017-0279-7

Zhang, J., Guo, J., Fang, W., Jun, Q., \& Shi, K. (2015). Clinical features of MELAS and its relation with A3243G gene point mutation. International Journal of Clinical and Experimental $\mathrm{Pa}$ thology, 8(10), 13411-5. [PMID] [PMCID] 